\title{
Childhood undernutrition in three disadvantaged East African Districts: a multinomial analysis
}

\author{
Kingsley E. Agho ${ }^{1 *}$, Blessing J. Akombi ${ }^{1}$, Akhi J. Ferdous ${ }^{2}$, Irene Mbugua ${ }^{3}$ and Joseph K. Kamara ${ }^{4}$
}

\begin{abstract}
Background: Undernutrition is an important public health indicator for monitoring nutritional status and survival. In spite of its importance, undernutrition is a significant problem health problem in many East African communities. The aim of this study was to identify factors associated with childhood undernutrition in three disadvantaged East African Districts.

Methods: We examined data for 9270 children aged 0-59 months using cross-sectional survey from Gicumbi District in Rwanda, Kitgum District in Uganda and Kilindi District in Tanzania. We considered the level of undernutrition (stunting, wasting and underweight) as the outcome variables with four ordinal categories (severely undernourished, moderately undernourished, mildly undernourished, and nourished). Generalized linear latent and mixed models (GLLAMM) with the mlogit link and binomial family that adjusted for clustering and sampling weights were used to identify factors associated with undernutrition among children aged 0-59 months in three disadvantaged East African Districts.

Results: After adjusting for potential confounding factors, the odds of a child being stunted were higher in Gicumbi District in Rwanda while the odds of a child being wasted and underweight were higher in Kitgum District in Uganda. Having diarrhoea two weeks prior to the survey was significantly associated with severe undernutrition. Wealth index (least poor household), increasing child's age, sex of the child (male) and unavailability of water all year were reported to be associated with moderate or severe stunting/wasting. Children of women who did not attend monthly child growth monitoring sessions and children who had Acute Respiratory Infection (ARI) symptoms were significantly associated with moderate or severe underweight.
\end{abstract}

Conclusions: Findings from our study indicated that having diarrhoea, having ARI, not having water availability all year and not attending monthly child growth monitoring sessions were associated with undernutrition among children aged 0-59 months. Interventions aimed at improving undernutrition in these disadvantaged communities should target all children especially those children from households with poor sanitation practices.

Keywords: Morbidity, Mortality, Multinomial, Malnutrition, Undernutrition, East Africa

\footnotetext{
* Correspondence: k.agho@westernsydney.edu.au

${ }^{1}$ School of Science and Health, Western Sydney University, Locked Bag 1797,

Penrith, NSW 2751, Australia

Full list of author information is available at the end of the article
}

(c) The Author(s). 2019 Open Access This article is distributed under the terms of the Creative Commons Attribution 4.0 International License (http://creativecommons.org/licenses/by/4.0/), which permits unrestricted use, distribution, and reproduction in any medium, provided you give appropriate credit to the original author(s) and the source, provide a link to the Creative Commons license, and indicate if changes were made. The Creative Commons Public Domain Dedication waiver (http://creativecommons.org/publicdomain/zero/1.0/) applies to the data made available in this article, unless otherwise stated. 


\section{Background}

Undernutrition is a major public health problem and an important health indicator for monitoring nutritional status and survival of children under- 5 years in many developing countries around the world [1]. Measures of childhood undernutrition are used to track development progress and socioeconomic inequalities in many low and middle-income countries. Suboptimal nutrition in the first 1000 days of life could lead to impaired physical development, which has a long-term impact on cognitive ability thus resulting in reduced educational performance and economic productivity in adulthood [2]. Undernutrition lowers immunity thereby predisposing a child to the higher risk of infections, as well as increases the frequency and severity of such infections, and also delay recovery. The relationship between undernutrition and infection creates a vicious cycle of worsening illness and deteriorating nutritional status as undernutrition can make a child more susceptible to infection, and infection also contributes to undernutrition [3].

Sub-Saharan Africa bears one of the highest burdens of undernutrition. In 2016, more than one-third of stunted children (38\%) and more than one-quarter of wasted (27\%) children lived in sub-Saharan Africa. However, a more detailed look into the distribution of undernutrition within sub-Saharan Africa shows that Eastern Africa (36.7\%) has a higher prevalence of stunting compared to Western Africa (21.4\%), Central Africa (32.5\%), and Southern Africa (28.1\%) [4]. While Western Africa $(8.5 \%)$ has a higher rate of wasting than Central Africa (7.3\%), Southern Africa (5.5\%), and Eastern Africa (6.5\%) [4], these estimates reveal regional disparities in the distribution of undernutrition, thus the need to identify region-specific factors contributing to the distribution of undernutrition across the regions.

The predictors of childhood undernutrition are well-researched and could be classified as either proximate or distal, and affect the nutritional status of children at different levels $[5,6]$. Proximate factors operate at the individual and child level which includes age, sex, birth size, birth order, birth interval and infections [7, 8]. The distal factors include a wider range of conceptual factors within the socio-cultural, economic, environmental, climatic and political context which influence food (in) security, sanitation, access to health care services and education at the household and community level [9]. Studies have reported that these factors tend to vary spatially depending on geographic location and climatic conditions. Previous studies have reported a relationship between undernutrition and geographical region, clearly acknowledging that a child's geographic location is an important modifier of known determinants of undernutrition [5, 7]. However, despite the huge body of evidence on the relationship between undernutrition and geographical region, there is limited evidence of analysis of the factors associated with childhood undernutrition across communities within highly burdened countries in the same sub-region in order to identify the most consistent factors. Given the high rate of undernutrition in Eastern Africa, this study aims to identify the specific factors associated with childhood undernutrition in three East African Districts in order to drive targeted interventions within these communities. With a decrease in undernutrition within communities, there will be a corresponding decrease at the national and sub-regional level thus setting Eastern Africa on the path to achieving the World Health Organization (WHO) global nutrition target by 2025 .

\section{Methods}

\section{Study area}

Gicumbi district is situated in the Northern Province of Rwanda and has a population of 395,606 residents. Gicumbi District covers 21 sectors, 109 cells, 630 villages (Imidugudu). It has 23 health centres, 1 district hospital and 1 prison clinic. Rwanda has a health development strategy based on decentralized management and district-level care [10]. Kilindi district is located in the northern zone of Tanzania with a population of 236,833 residents. Kilindi District comprises of 16 rural wards and 102 villages. It has 30 dispensaries, 3 health centres and one hospital [11]. Tanzania has a hierarchical health system made up of the dispensaries found in every village, health centres at the ward level, district hospital at the district level, the regional referral hospital at the regional level, the zone hospitals at the tertiary level and the national hospital at the national level. There are also some specialized hospitals which do not fit directly into this hierarchy and therefore are directly linked to the ministry of health [12]. Kitgum district is located in the northern region of Uganda and has a population of 247,800 residents. It comprises 51 parishes and 437 village councils [13]. Kitgum district has 2 hospitals and 23 health centres; 21 are government owned while 4 are owned by non -government Organizations. Health services delivery in Uganda is decentralized within national, districts and health sub-districts levels with referral hospitals at the national level and health centres at the district and sub-district levels [14].

The sample was in two stages. In the first stage, a total of 20 villages (clusters) were selected from cells for Gicumbi, wards for Kilindi and Parishes for Kitgum. In the second stage, 32 households were randomly selected in each selected villages (clusters). The detailed sampling procedure for Gicumbi in Rwanda has been reported elsewhere [15]. For district-level results, sample weights will be used, and sampling weight was calculated by the product of the reciprocal of the sampling fractions employed in the selection of (cells for Gicumbi, wards 
for Kilindi and Parishes for Kitgum). For the combined analysis of the three datasets, we re-normalised our sampling weights by computing the total sum of weights for each district and divide each district survey sampling weights with the total sum of weights.

\section{Data source}

Our dataset was obtained from a survey conducted during the harvest period, from 21st- 31st of January, 2016 in Gicumbi district in Rwanda, Kitgum district in Uganda and Kilindi district in Tanzania. The survey was commissioned as part of World Vision Rwanda, Uganda and Tanzania funding service agreement to generate evidence to influence maternal and child health programmes which aimed to reach 36,250 disadvantaged beneficiaries in these East African districts. The Maternal Newborn Child Health $(\mathrm{MNCH})$ Project aimed to collect health and related indicators to identify the health needs of women and children and to establish priorities for evidence-based planning, decision-making in these regions. The program was an opportunity for World Vision to embed knowledge and action of the organisation's '7-11' interventions for maternal and child survival in the Region [16]. World Vision uses the 7-11 approach to prevent maternal and child mortality and morbidity through 7 key interventions for a mother and 11 interventions for the child. The intervention for the mother are: diet, deworming and iron supplements, prevention of infectious diseases, malaria prevention and treatment, appropriate pregnancy spacing, birth preparedness, and access to antenatal and postnatal maternity services. The 11 interventions for the child are appropriate breastfeeding, newborn care, timely complementary feeding, age-appropriate immunisation, sufficient iron intake, consistent hand washing prevention and treatment for acute malnutrition, prevention and treatment of malaria, and acute respiratory infection. Others are timely administration of oral rehydration therapy to treat diarrhoea, prevention and care for pediatric Human Immunodeficiency Virus (HIV), and timely deworming [17].

\section{Study outcomes}

The nutritional status of children under five years of age was measured anthropometrically. We considered height-for-age (stunting), weight-for-height (wasting) and weight-for-age (underweight). The height-for-age index is an indicator of linear growth retardation and cumulative growth deficits in children, Weight-for-height index measures body mass in relation to height and reflects the current nutritional status of the child. Weight-for-age takes into account both acute malnutrition (wasting) and chronic malnutrition (stunting), but it does not distinguish between stunting and wasting. The index is calculated using growth standards published by WHO in 2006 . These growth standards were generated through data collected in the WHO Multicentre Growth Reference Study and expressed in standard deviation units from the Multicentre Growth Reference Study median [18]. Child undernutrition status was categorized into four categories - severe undernutrition ( $<-3.0 \mathrm{Z}$-score), moderate undernutrition ( -3.0 to $-2.0 \mathrm{Z}$-score) and mild undernutrition $(-2.0$ to $-1.0 \mathrm{Z}$-score $)$ and proper nutrition $(\geq-1.0$ Z-score). The level of childhood undernutrition (stunting, wasting and underweight) was considered as the outcome variables with four ordinal categories (severely undernourished, moderately undernourished, mildly undernourished, and nourished).

\section{Potential confounders}

The potential confounding factors were organised into four distinct groups: Socio-economic and demographic (Districts, primary caregiver, education level, marital status, household wealth index and food security); child (fever in two weeks preceding each survey, acute respiratory infection (ARI) in two weeks prior to each survey, diarrhoea in the two weeks preceding each survey, sex of baby and child's age in months); maternal and child health (antenatal care and attended child monthly growth monitoring sessions); health services and environmental factors (quality of care from health services, place of delivery, water available all year, sources of drinking water and type of toilet facility).

The household wealth index variable measures basic household needs for all children 5-18 years. The household wealth index was constructed by assigning weights to three basic household needs for children 5-18 years (i.e. difficulty providing at least two sets of clothes for all children aged 5-18 years living in the household, difficulty providing a pair of shoes for all children aged 518 years living in the household and difficulty paying school fees or school contribution for all children aged 5-18 years living in the household) using principal components analysis. The household wealth index was divided into three categories: poorest, middle and least poor [19]. Improved and unimproved sources of drinking water and type of toilet facility were categorised based on the WHO and UNICEF Joint Monitoring Programme guidelines [20]. Birth order and number of children per household were not collected. Higher birth order and number of children per household may result in higher susceptibility to undernutrition due to impact on income.

\section{Statistical analysis}

Re-normalised weight was used for Survey (SVY) tabulation that adjusts for clustering, and sampling weights were used to determine the percentage and frequency 
count of all selected characteristics and, district-specific weights were used for the Taylor series linearization method in the surveys when estimating 95\% confidence intervals around prevalence estimates of undernutrition by severity in each district.

Multivariate multinomial logistic regression model guided by the conceptual framework was used to determine factors associated with childhood undernutrition (stunting, wasting and underweight) with nourished children used as reference category.

In the multivariate analyses, a four-stage model was carried out, in the first stage model, socio-economic and demographic factors were entered into the model, and a stepwise backward elimination method was used to remove the non-significant factors $(p>0.05)$. In the second stage model, the significant factors in the first stage model were added to the child level factors, and this was followed by another stepwise backward elimination procedure which retained all the significant factors. A similar procedure was employed for the third stage model which included the individual (maternal and child) level factors as well as health services factors and the final stage model which introduced environmental factors. After completion of all four modelling stages, the factors that were significantly associated with the outcomes were retained. All statistical analyses were conducted using STATA/MP Version.14.1 (StataCorp, College Station, Texas, USA) and adjusted odds ratios (AORs) and their 95\% confidence intervals (CIs) obtained from the adjusted multivariate multinomial logistic regression model were used to measure the factors associated with childhood undernutrition.

\section{Results}

Distribution of children aged 0-59 months in three East African Districts is presented in Table 1. The majority of women were from Kitgum district in Uganda, and the proportion of respondents who were educated up to secondary level was about $9 \%$. Women who were never married made up $57 \%$ while $89 \%$ of the women reported being the primary caregiver.

The proportion of male and female children was relatively constant, and $51.2 \%$ of respondents were from poorest households. Over two-thirds of women reported having water availability all year and over $80 \%$ had no diarrhoea or ARI. The proportion of mildly, moderately and severely stunted were $25.1,16.8$ and $11.8 \%$, respectively while a high proportion of children reported either not wasted or not underweight. Percentage distribution of childhood undernutrition aggregated by three East African Districts was reported Additional file 1: Table S1.

\section{Prevalence of undernutrition}

Figures 1, 2 and 3 show the prevalence and 95\% confidence Intervals of undernutrition (stunting, wasting and underweight) by severity. In the figures, if the $95 \%$ confidence intervals overlap implies non-significant different at the 95\% confidence level. In Fig. 1, the prevalence of severe and moderate stunting was significantly higher in Gicumbi district and Kilindi district but lower in Kitgum district while the prevalence of mild stunting was higher in Kitgum district compared with the other two districts (Gicumbi and Kilindi).

In Fig. 2, the prevalence of mild, moderate and severe wasting was significantly higher in Kitgum district compared with Gicumbi and Kilindi districts while the prevalence of underweight was also higher in Kitgum district than the other two districts (Gicumbi and Kilindi) as represented in Fig. 3.

\section{Factors associated with mild, moderate and severe stunting}

Table 2 shows the factors associated with mild, moderate and severe stunting in children aged $0-59$ months in three disadvantaged East African districts. The odds of a child being moderately or severely stunted significantly decreased in Kitgum and Kilindi districts compared to Gicumbi district. Significantly increased odds of mild, moderate and severe stunting were reported among household wealth index (least poor), primary caregiver (mothers), male child, child aged 24-59 months and children who reported having diarrhoea.

\section{Factors associated with mild, moderate and severe wasting}

Table 3 shows the factors associated with mild, moderate and severe wasting in children aged 0-59 months in three disadvantaged East African districts. Mild, moderate and severe wasting significantly increased in Kitgum district compared with Kilindi and Gicumbi districts. Significantly increased odds of mild and severe wasting were observed among the middle household wealth index while significantly increased odds of moderate and severe wasting were reported among the least poor. Maternal education (no schooling), primary giver (mothers), male child, no water availability all year and children who reported having diarrhoea were significantly associated with mild, moderate and severe wasting. The odds of moderate and severe wasting were significantly higher in children aged 24-59 months while the source of drinking water (unimproved) was significantly associated with mild wasting.

\section{Factors associated with mild, moderate and severe underweight}

Table 4 shows the factors associated with mild, moderate and severe underweight in children aged 0-59 months in three disadvantaged East African districts. The odds of a child being mildly, moderately or severely underweight 
Table 1 Distribution of children aged 0-59 months in three disadvantaged East African Districts $(n=9270)$

\begin{tabular}{lll}
\hline Variables & $\mathrm{n}$ & Percent (\%) \\
\hline
\end{tabular}

Socio-economic and demographic factors

District (Country)

Gicumbi (Rwanda)

2349

25.3

Kitgum (Uganda)

4267

46.0

Kilindi (Tanzania)

2654

28.6

Primary caregiver

Mother

8261

89.1

Others

1009

10.9

Education level $(n=9266)$

No schooling

3864

41.7

Primary

4590

49.5

Secondary and Tertiary

812

8.8

Marital status ( $n=9266)$

Never married

5268

56.8

Currently married

3789

40.9

Formerly married

209

2.3

Household wealth index

Poorest

4744

Middle

Least Poor

51.2

29.3

1813

19.6

Child factors

Sex of child

$\begin{array}{lll}\text { Male } & 4552 & 49.1 \\ \text { Female } & 4718 & 50.9 \\ \text { Child's Age in months } & & \\ \text { 0-23 months } & 6295 & 67.9 \\ \text { 24-59 months } & 2975 & 32.1\end{array}$

Maternal and child's health factors

Antenatal care (ANC, $n=8904$ )

Inadequate ( $<4$ visits)

2374

25.6

Adequate (4+ visits)

6530

70.4

Attended child monthly growth monitoring sessions ( $n=8163$ )

Yes

6941

74.9

No

1722

18.6

Health services and Environmental factors

Quality of care from health services $(n=8170)$

Very good

1379

Good

4857

52.4

Not good

1934

20.9

Place of delivery $(n=9266)$

Government health unit

7003

75.5

Others

2263
Table 1 Distribution of children aged 0-59 months in three disadvantaged East African Districts $(n=9270)$ (Continued)

\begin{tabular}{|c|c|c|}
\hline Variables & $n$ & Percent (\%) \\
\hline \multicolumn{3}{|c|}{ Water availability all year } \\
\hline Yes & 7071 & 76.3 \\
\hline No & 2199 & 23.7 \\
\hline \multicolumn{3}{|c|}{ Sources of drinking water } \\
\hline Improved & 5954 & 64.2 \\
\hline Unimproved & 3316 & 35.8 \\
\hline \multicolumn{3}{|l|}{ Type of toilet facility } \\
\hline Improved & 469 & 5.1 \\
\hline Unimproved & 8801 & 94.9 \\
\hline \multicolumn{3}{|c|}{ Household food security } \\
\hline Food security & 1494 & 16.1 \\
\hline Mild & 361 & 3.9 \\
\hline Moderate & 3950 & 42.6 \\
\hline Food insecurity & 3465 & 37.4 \\
\hline \multicolumn{3}{|l|}{ Fever } \\
\hline No Fever & 6003 & 64.8 \\
\hline Had Fever & 3267 & 35.2 \\
\hline \multicolumn{3}{|l|}{ Diarrhoea } \\
\hline No diarrhoea & 7802 & 84.2 \\
\hline Had diarrhoea & 1468 & 15.8 \\
\hline \multicolumn{3}{|l|}{ ARI } \\
\hline No ARI & 7786 & 84.0 \\
\hline Had ARI & 1484 & 16.0 \\
\hline
\end{tabular}

Child undernutrition status

Stunting $(n=8706)$

$\begin{array}{lll}\text { Not stunted } & 3516 & 37.9 \\ \text { Mildly stunted } & 2405 & 25.1 \\ \text { Moderately stunted } & 1631 & 16.8 \\ \text { Severely stunted } & 1154 & 11.8 \\ \text { Wasting }(n=8706) & & \\ \text { Not wasted } & 6067 & 65.4 \\ \text { Mildly wasted } & 1917 & 20.7 \\ \text { Moderately wasted } & 572 & 6.2 \\ \text { Severely wasted } & 150 & 1.6\end{array}$

Underweight $(n=8706)$

$\begin{array}{lll}\text { Not underweight } & 7264 & 70.4\end{array}$

Mildly underweight $\quad 985 \quad 10.6$

Moderately underweight $\quad 321 \quad 3.5$

Severely underweight $\quad 138 \quad 1.5$

Acute respiratory infection (ARI)

Weighted total $=9270$ unless otherwise given in parenthesis

were significantly higher in Kitgum district compared with Kilindi and Gicumbi districts. Increased odds of mild, moderate and severe wasting were observed 


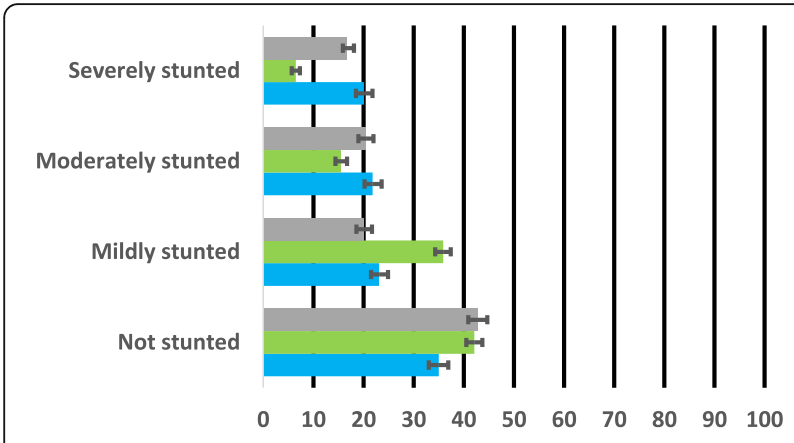

- Kilindi (Tanzania) Kitgum (Uganda) Gicumbi (Rwanda)

Fig. 1 Prevalence and 95\% confidence intervals (Cls) of stunting by severity

among mother of children who did not attend child monthly growth monitoring sessions, a child aged 0-23 months and children who reported having diarrhoea. Children who had ARI symptoms were 1.18 times and 1.82 times more likely to report higher odds of moderately or severely underweight, respectively.

\section{Discussion}

This study identified the factors associated with childhood undernutrition in three East African Districts. The main factors associated with moderate or severe stunting/wasting were: wealth index (poorest households), increasing child's age, sex of the child (male) and unavailability of water all year. Children of women who did not attend monthly child growth monitoring sessions and children who had ARI symptoms were significantly associated with moderate or severe underweight while having diarrhoea two weeks prior to the survey was significantly associated with severe undernutrition (stunting, wasting and underweight).

In this study, children under-five years residing in Gicumbi District in Rwanda were more predisposed to

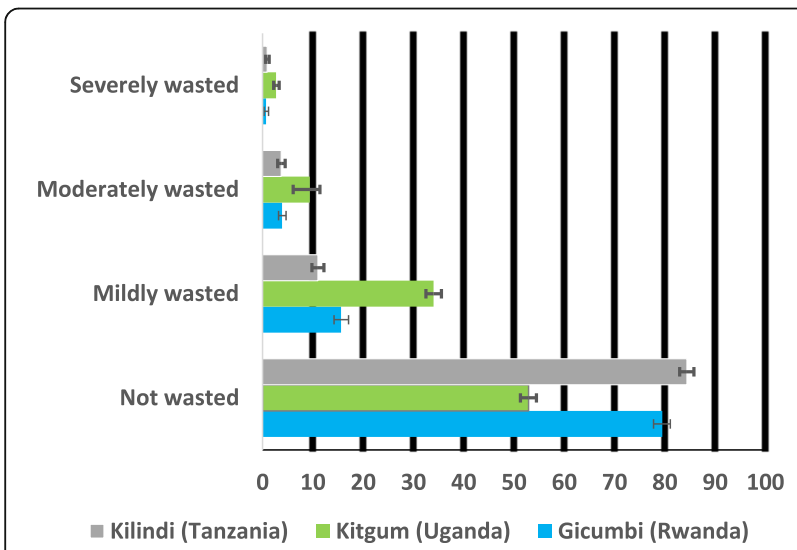

Fig. 2 Prevalence and 95\% confidence intervals (Cls) of wasting by severity

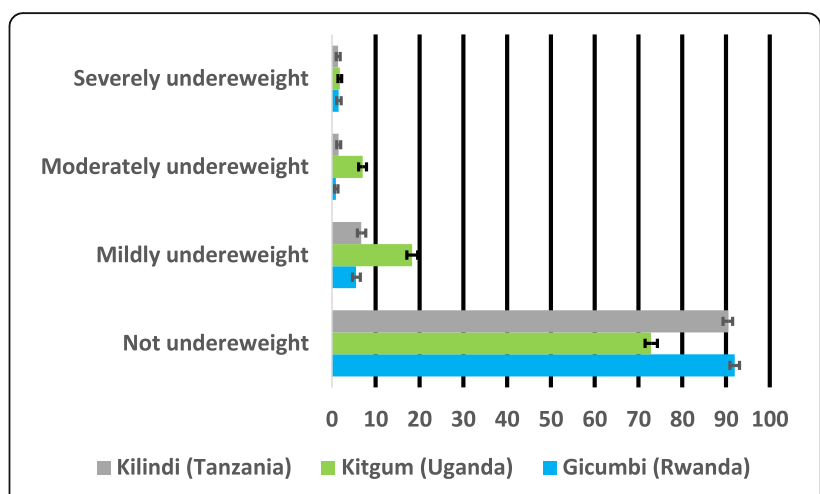

Fig. 3 Prevalence and 95\% confidence intervals (Cls) of underweight by severity

stunting while the odds of a child being wasted and underweight were higher in Kitgum District in Uganda. Though all three districts analysed were in East Africa, disparities still existed in the distribution of child undernutrition, and this might be due to specific features associated with the district's geographic location. This report shows that geographic location may influence child nutrition and is a determinant of childhood undernutrition. Geographic location affects the cultivation of food crops, which in turn affects access and availability of food for household consumption and sale. The impact of geographical location could be seen in the environmental variability and predominant occupation practised in the region which could influence food (in) security and consequently affect child nutrition, growth and development $[21,22]$. Geographic locations with more favourable climatic conditions and whose residents are predominantly farmers have greater access to variable food types and are more food secured [21]. However, studies have shown that cultural beliefs and practices unique to a region influence the food given to the growing child despite its overall nutritional value and availability $[6,7,23]$.

In this study, the wealth index was used as a proxy to access socioeconomic status. Children from poor households were more susceptible to childhood stunting and wasting when compared to their counterparts from richer households in all three East African Districts. Evidence from previously conducted studies shows the inverse relationship between socioeconomic status and childhood stunting/wasting [6, 24-26]. These studies confirm that children from households with low socioeconomic status lack access to sufficient food of adequate quality, basic health care services, and have a higher risk of infection as a result of compromised immunity due to poor nutrition and suboptimal living conditions [27].

Child's age was also reported as a major factor associated with stunting and wasting in the study area. Suboptimal growth increased with age as older children 
Table 2 Factors associated with mildly, moderately and severely stunted in children aged 0-59 months in three disadvantaged East African Districts

\begin{tabular}{|c|c|c|c|c|c|c|}
\hline \multirow[t]{2}{*}{ Variables } & \multicolumn{2}{|l|}{ Mildly stunted } & \multicolumn{2}{|c|}{ Moderately Stunted } & \multicolumn{2}{|c|}{ Severely stunted } \\
\hline & AOR $(95 \% \mathrm{Cl})$ & $P$-value & AOR $(95 \% \mathrm{Cl})$ & P-value & AOR (95\%Cl) & $P$-value \\
\hline \multicolumn{7}{|l|}{ District (Country) } \\
\hline Gicumbi (Rwanda) & 1.00 & & 1.00 & & 1.00 & \\
\hline Kitgum (Uganda) & $1.10(0.94,1.29)$ & 0.227 & $0.47(0.40,0.57)$ & $<0.001$ & $0.18(0.15,0.23)$ & $<0.001$ \\
\hline Kilindi (Tanzania) & $0.92(0.76,1.10)$ & 0.342 & $0.93(0.77,1.1)$ & 0.495 & $0.67(0.52,0.82)$ & $<0.001$ \\
\hline \multicolumn{7}{|c|}{ Household wealth index } \\
\hline Poorest & 1.00 & & 1.00 & & 1.00 & \\
\hline Middle & $1.09(0.95,1.26)$ & 0.209 & $1.07(0.91,1.26)$ & 0.397 & $1.04(0.86,1.26)$ & 0.679 \\
\hline Least Poor & $1.37(1.18,1.59)$ & $<0.001$ & $1.30(1.09,1.5)$ & 0.003 & $1.36(1.11,1.67)$ & 0.003 \\
\hline \multicolumn{7}{|l|}{ Primary caregiver } \\
\hline Mother & 1.00 & & 1.00 & & 1.00 & \\
\hline Others & $0.85(0.72,1.00)$ & 0.056 & $0.73(0.59,0.91)$ & 0.005 & $0.45(0.32,0.64)$ & $<0.001$ \\
\hline \multicolumn{7}{|l|}{ Sex of the baby } \\
\hline Male & 1.00 & & 1.00 & & 1.00 & \\
\hline Female & $0.81(0.73,0.90)$ & $<0.001$ & $0.69(0.61,0.78)$ & $<0.001$ & $0.54(0.47,0.62)$ & $<0.001$ \\
\hline \multicolumn{7}{|l|}{ Child's Age in months } \\
\hline 0-23 months & 1.00 & & 1.00 & & 1.00 & \\
\hline 24-59 months & $2.18(1.91,2.49)$ & $<0.001$ & $2.53(2.16,2.97)$ & $<0.001$ & $2.45(2.03,2.96)$ & $<0.001$ \\
\hline \multicolumn{7}{|l|}{ Diarrhoea } \\
\hline No diarrhoea & 1.00 & & 1.00 & & 1.00 & \\
\hline Had diarrhoea & $1.13(0.97,1.31)$ & 0.126 & $1.28(1.07,1.53)$ & 0.008 & $1.80(1.45,2.33)$ & $<0.001$ \\
\hline
\end{tabular}

Confounding factors adjusted for are: Socio-economic and demographic factors; child factors; child undernutrition status, maternal, health services and environmental factors and child's health factors

were reported to be more predisposed to childhood stunting/wasting. This increase in child undernutrition with age could be as a result of an increase in energy expenditure by the older child without sufficient and adequate food intake. It could also result from an increased interaction of the older child with its immediate environment which may lead to increased risk of infections and exposure to childhood diseases either through drinking water from unimproved sources, consumption of contaminated foods, poor hygiene or poor environmental sanitation [27]. Furthermore, as the child's age increases, s/he requires more energy (calories) and nutrients for proper growth and development. The inability of a growing child to meet required daily energy and nutrient needs could result in undernourishment [7].

Male children were more prone to childhood stunting/ wasting than their female counterparts. Male children tend to be more physically active thereby expending large amounts of energy which should be channelled towards proper growth and development. This finding is consistent with results from other studies carried out in Nigeria [6, 7], Iran [24], Kenya [28], Indonesia [29], Tanzania [30] Ghana [21], Ethiopia [31] and South
Africa [32]. The studies reported a higher prevalence of childhood undernutrition among male children than females. However, a biological reason for this is still unknown.

Unavailability of water was reported as a significant factor associated with moderate and severe stunting. Lack of access to clean and safe water from improved water sources could have a significant detrimental effect on child growth and development resulting from sustained exposure to enteric pathogens and diarrhoeal disease [33]. Thus, improvements in the quantity and microbial quality of water available to the growing child is essential if the child is to meet its nutritional needs and reduce the risk of infection or dehydration. Additionally, the unavailability of water for irrigation purposes affects farming leading to low food production and food insecurity especially in locations with low rainfall. Household unavailability of water may result from the inability of households with low socio-economic status to afford the cost of clean drinking water. Therefore, to improve access to water and the nutritional status of children, consistent access to affordable, clean water, especially in disadvantaged communities, is vital [33]. 
Table 3 Factors associated with mildly, moderately and severely wasted in children aged 0-59 months in three disadvantaged East African Districts

\begin{tabular}{|c|c|c|c|c|c|c|}
\hline \multirow[t]{2}{*}{ Variables } & \multicolumn{2}{|l|}{ Mildly wasted } & \multicolumn{2}{|c|}{ Moderately wasted } & \multicolumn{2}{|l|}{ Severely wasted } \\
\hline & AOR $(95 \% \mathrm{Cl})$ & $P$-value & AOR $(95 \% \mathrm{Cl})$ & $P$-value & AOR $(95 \% \mathrm{Cl})$ & $P$-value \\
\hline \multicolumn{7}{|l|}{ District (Country) } \\
\hline Gicumbi (Rwanda) & 1 & & 1 & & 1 & \\
\hline Kitgum (Uganda) & $3.90(3.28,4.65)$ & $<0.001$ & $2.99(2.22,4.04)$ & $<0.001$ & $1.94(1.11,3.39)$ & 0.021 \\
\hline Kilindi (Tanzania) & $0.66(0.54,0.80)$ & $<0.001$ & $0.96(0.68,1.36)$ & 0.824 & $0.46(0.2,0.89)$ & 0.022 \\
\hline \multicolumn{7}{|c|}{ Maternal education level } \\
\hline No schooling & 1 & & 1 & & 1 & \\
\hline Primary & $0.91(0.81,1.02)$ & 0.096 & $0.91(0.81,1.02)$ & 0.346 & $0.70(0.9,0.99)$ & 0.043 \\
\hline Secondary+ & $0.99(0.82,1.21)$ & 0.970 & $0.75(0.53,1.06)$ & 0.101 & $0.77(0.43,1.41)$ & 0.410 \\
\hline \multicolumn{7}{|c|}{ Household wealth index } \\
\hline Poorest & 1 & & 1 & & 1 & \\
\hline Middle & $1.25(1.08,1.44)$ & 0.002 & $1.02(0.80,1.29)$ & 0.900 & $2.98(1.96,4.53)$ & $<0.001$ \\
\hline Least Poor & $1.02(0.88,1.18)$ & 0.816 & $1.43(1.14,1.79)$ & 0.002 & $1.59(1.00,2.53)$ & 0.048 \\
\hline \multicolumn{7}{|l|}{ Primary caregiver } \\
\hline Mother & 1 & & 1 & & 1 & \\
\hline Others & $0.96(0.81,1.14)$ & 0.651 & $1.00(0.77,1.30)$ & 0.992 & $0.36(0.19,0.67)$ & 0.001 \\
\hline \multicolumn{7}{|l|}{ Sex of the baby } \\
\hline Male & 1 & & 1 & & 1 & \\
\hline Female & $0.86(0.77,0.96)$ & 0.006 & $0.84(0.71,1.00)$ & 0.055 & $0.61(0.44,0.86)$ & 0.004 \\
\hline \multicolumn{7}{|c|}{ Water availability all year } \\
\hline No & 1 & & 1 & & 1 & \\
\hline Yes & $0.77(0.67,0.89)$ & $<0.001$ & $0.75(0.60,0.95)$ & 0.016 & $0.41(0.24,0.68)$ & 0.001 \\
\hline \multicolumn{7}{|l|}{ Child's Age in months } \\
\hline 0-23 months & 1 & & 1 & & 1 & \\
\hline 24-59 months & $0.93(0.82,1.06)$ & 0.279 & $1.23(1.00,1.52)$ & 0.047 & $1.48(1.00,2.18)$ & 0.048 \\
\hline \multicolumn{7}{|c|}{ Sources of drinking water } \\
\hline Improved & 1 & & 1 & & 1 & \\
\hline Unimproved & $1.30(1.13,1.50)$ & $<0.001$ & $0.95(0.75,1.22)$ & 0.701 & $0.42(0.25,0.71)$ & 0.001 \\
\hline \multicolumn{7}{|l|}{ Diarrhoea } \\
\hline No diarrhoea & 1 & & 1 & & 1 & \\
\hline Had diarrhoea & $1.18(1.02,1.37)$ & 0.030 & $2.26(1.83,2.80)$ & $<0.001$ & $3.66(2.51,5.32)$ & $<0.001$ \\
\hline
\end{tabular}

Confounding factors adjusted for are: Socio-economic and demographic factors; child factors; child undernutrition status, maternal, health services and environmental factors and child's health factors

Having diarrhoeal episodes and manifesting ARI symptoms two weeks prior to the survey were significantly associated with severe undernutrition. Studies have confirmed the relationship between ARI, diarrhoea and undernutrition which results in a vicious cycle [34-38]. Gastrointestinal and respiratory infections reduce appetite, increases catabolism and inhibit intestinal absorption of nutrients from food thus leading to increased susceptibility to severe undernutrition especially underweight [36, 37]. Consequently, child undernutrition leads to immune dysfunction, such as the impairment of cell-mediated immunity, cytokine and immunoglobulin production which lowers immunity and predisposes a child to infectious diseases [36, 38, 39]. Diarrhoea and ARI often occur due to suboptimal child feeding practices and an unhealthy environment [40]. Studies have also shown that exclusive breastfeeding in the first 6 months of life and the timely introduction of appropriate complementary foods (6-23 months) reduces the risk of diarrhoea and ARI [36, 37]. Proper child feeding practices build the child's immunity through the transfer of innate immune components such as secretory IgA, lactoferrin and lysozyme from mother to child during breastfeeding and enhance antibody response to 
Table 4 Factors associated with mildly, moderately and severely underweights in children aged 0-59 months in three disadvantaged East African Districts

\begin{tabular}{|c|c|c|c|c|c|c|}
\hline \multirow[t]{2}{*}{ Variables } & \multicolumn{2}{|c|}{ Mildly underweight } & \multicolumn{2}{|c|}{ Moderately underweight } & \multicolumn{2}{|c|}{ Severely underweight } \\
\hline & AOR $(95 \% \mathrm{Cl})$ & $P$-value & AOR $(95 \% \mathrm{Cl})$ & $P$-value & AOR $(95 \% \mathrm{Cl})$ & $P$-value \\
\hline \multicolumn{7}{|l|}{ District (Country) } \\
\hline Gicumbi (Rwanda) & 1 & & 1 & & 1 & \\
\hline Kitgum (Uganda) & $5.66(4.48,7.19)$ & $<0.001$ & $11.64(7.09,19.11)$ & $<0.001$ & $1.11(0.60,2.07)$ & 0.741 \\
\hline Kilindi (Tanzania) & $1.00(0.76,1.32)$ & 0.997 & $1.93(1.08,3.47)$ & 0.027 & $0.20(0.10,0.40)$ & $<0.001$ \\
\hline \multicolumn{7}{|c|}{ Household wealth index } \\
\hline Poorest & 1 & & 1 & & 1 & \\
\hline Middle & $0.92(0.77,1.11)$ & 0.393 & $0.47(0.33,0.67)$ & $<0.001$ & $1.65(1.03,2.66)$ & 0.038 \\
\hline Least Poor & $0.89(0.74,1.07)$ & 0.211 & $1.25(0.95,1.64)$ & 0.107 & $1.48(0.92,2.35)$ & 0.103 \\
\hline \multicolumn{7}{|l|}{ Sex of the baby } \\
\hline Male & 1 & & 1 & & 1 & \\
\hline Female & $1.30(1.12,1.50)$ & $<0.001$ & $0.75(0.59,0.95)$ & 0.018 & $1.31(0.90,1.91)$ & 0.154 \\
\hline \multicolumn{7}{|c|}{ Attended child monthly growth monitoring sessions } \\
\hline Yes & 1 & & 1 & & 1 & \\
\hline No & $1.11(0.93,1.32)$ & 0.232 & $1.33(1.02,1.74)$ & 0.036 & $2.19(1.44,3.35)$ & $<0.001$ \\
\hline \multicolumn{7}{|l|}{ Child's Age in months } \\
\hline 0-23 months & 1 & & 1 & & 1 & \\
\hline 24-59 months & $0.50(0.43,0.59)$ & $<0.001$ & $0.39(0.30,0.51)$ & $<0.001$ & $0.30(0.18,0.49)$ & $<0.001$ \\
\hline \multicolumn{7}{|c|}{ Sources of drinking water } \\
\hline Improved & 1 & & 1 & & 1 & \\
\hline Unimproved & $1.12(0.92,1.36)$ & 0.267 & $0.94(0.65,1.35)$ & 0.727 & $3.28(2.07,5.19)$ & $<0.001$ \\
\hline \multicolumn{7}{|l|}{ Type of toilet facility } \\
\hline Improved & 1 & & 1 & & 1 & \\
\hline Unimproved & $2.29(1.37,3.85)$ & 0.002 & $1.42(0.73,2.75)$ & 0.302 & $0.59(0.27,1.31)$ & 0.198 \\
\hline \multicolumn{7}{|l|}{ Diarrhoea } \\
\hline No diarrhoea & 1 & & 1 & & 1 & \\
\hline Had diarrhoea & $1.21(1.01,1.44)$ & 0.044 & $1.17(0.87,1.54)$ & 0.267 & $8.90(5.59,14.17)$ & $<0.001$ \\
\hline \multicolumn{7}{|l|}{ ARI } \\
\hline No ARI & 1.00 & & 1.00 & & 1.00 & \\
\hline Had ARI & $0.69(0.56,0.84)$ & $<0.001$ & $1.18(0.90,1.56)$ & 0.235 & $1.87(1.19,2.91)$ & 0.006 \\
\hline
\end{tabular}

Confounding factors adjusted for are: Socio-economic and demographic factors; child factors; child undernutrition status, maternal, health services and environmental factors and child's health factors

pathogens thus preventing infections [36, 37]. Therefore, to prevent ARI and the occurrence of diarrhoea, interventions targeted at promoting exclusive breastfeeding, vaccination, Los-osmolarity ORS, zinc and Vitamin A supplementation, availability of safe water, improved sanitation practices and preventing household pollution should be effected [40].

Children of women who did not attend monthly child growth monitoring sessions were significantly associated with moderate or severe underweight. Attending monthly child growth monitoring sessions allows for the evaluation of child growth in order to assess the appropriateness of the child's nutrient intake and sanitation.
Additionally, the high prevalence of undernutrition in children under- 5 years reported in this study may be attributed to the main lean period (October-December) before the survey when food prices are unreasonably high due to seasonal variability, and this continues up to harvest period before gradually decreasing.

\section{Strengths and limitations}

This study had several strengths. First, the study was population-based with a large sample size which is representative of the study area. Second, it applied appropriate statistical adjustments to data obtained from the survey and identified the most vulnerable 
subpopulation affected by childhood undernutrition in a large sample. However, this study also had some limitations. First, due to the cross-sectional nature of the study design, a causal relationship between the observed risk factors and childhood undernutrition cannot be established. Second, this study did not include validity assessments of undernutrition; no actual dietary assessments were made. Third, despite the use of a comprehensive set of variables in the analysis, the effect of genetics, birth defects, and early food allergies as well as the effect of residual confounding as a result of unmeasured covariates were not addressed. Finally, the measure of household wealth index using a small number of variables may misrepresent household expenditure and the actually socioeconomic status of the household.

\section{Policy implications}

Findings from this study are useful for public health planning to improve the nutritional status of children under-five years in East Africa. Intervention strategies geared towards reducing undernutrition in East African communities should focus on children from poor households with suboptimal sanitation practices. Communitybased educational sessions which educate women on the benefits of attending postnatal monthly child growth monitoring sessions should also be initiated.

\section{Conclusions}

Findings from our study indicate that having diarrhoea, ARI, unavailability of water supply all year and failure to attend monthly child growth monitoring sessions increases the odds of childhood undernutrition, especially among socioeconomically disadvantaged households. Thus interventions to reduce childhood undernutrition should focus on improving household sanitation and access to adequate water supply, as well as initiating community-based educational campaigns on the merits of attending child growth monitoring sessions. These interventions will improve the nutritional status of children under-five years in these East African communities which will invariably result in an overall decline in childhood undernutrition in Sub-Saharan Africa hereby setting the region on the path to achieving the WHO global nutrition target by 2025 .

\section{Additional file}

Additional file 1: Table S1: Percentage distribution of undernutrition in children aged 0-59 months by three East African Districts $(N=8,706)$. (DOCX $15 \mathrm{~kb}$ )

\section{Abbreviations}

ANC: Antenatal Care; AOR: Adjusted Odd Ratios; ARI: Acute Respiratory Infection; Cls: Confidence Intervals; HIV: Human Immunodeficiency Virus;
MNCH: Maternal Newborn Child Health; UNICEF: United Nations Children's Fund; WHO: World Health Organization

\section{Acknowledgements}

The authors would like to acknowledge the support of the district Local Government of Gicumbi, Kilindi and Kitgum, where these projects were implemented, the community leaders including Community Health Workers in identifying and mobilising eligible households and the parents who responded to the questions (mothers) and allowed the team to take anthropometric measurements on their children.

\section{Funding}

This study was part of end line evaluation for the East Africa maternal, newborn child health project. This project was funded by the Department of Foreign Affairs and Trade of the Australian Government through (AusAID Funding Order 62/37923 under the Australia Africa Community Engagement Scheme (AACES).

\section{Availability of data and materials}

The dataset analysed in this study is available as an additional supporting file.

\section{Authors' contributions}

KEA was involved in study conceptualization, data analysis and drafting of the manuscript. BJA was involved in drafting, editing and revising of the manuscript. JKK revised and enhanced the intellectual content of the manuscript. FJA revised the manuscript and made important contributions. IM was involved in editing and enhancing the intellectual content of the manuscript. All authors read and approved the final manuscript.

\section{Ethics approval and consent to participate}

Ethical clearance was obtained from Ministry of Health in Kigali, Kampala and Dar es Salaam, and necessary permission was also obtained from the Gicumbi, Kilindi and Kitgum regional health office and the Gicumbi, Kilindi and Kitgum health office and local administrators. Participants were given informed consent to sign before taking part in the survey, including assurance of anonymity and a description of how the data would be used. For illiterate participants, informed consent information was read aloud and signed. Mothers and children with serious illness were referred to the nearby health facilities. The data in this article presented as aggregate to ensure all respondents' identification information is obscured.

\section{Consent for publication}

Not applicable.

\section{Competing interests}

The authors declare that they have no competing interests.

\section{Publisher's Note}

Springer Nature remains neutral with regard to jurisdictional claims in published maps and institutional affiliations.

\section{Author details}

${ }^{1}$ School of Science and Health, Western Sydney University, Locked Bag 1797, Penrith, NSW 2751, Australia. ${ }^{2}$ The International Centre for Diarrhoeal Disease Research, Dhaka, Bangladesh. ${ }^{3}$ World Vision International, Karen Road, Off Ngong Road, P.O. Box 133, Karen, Nairobi 00502, Kenya. ${ }^{4}$ World Vision International, Southern Africa Regional Office, H100, Mbabane, Swaziland.

Received: 2 May 2018 Accepted: 1 April 2019

Published online: 23 April 2019

\section{References}

1. UNICEF. State of the World's Children Statistical Report. 2016.

2. Stewart CP, lannotti L, Dewey KG, Michaelsen KF, Onyango AW. Contextualising complementary feeding in a broader framework for stunting prevention. Matern Child Nutr. 2013;9(Suppl 2):27-45.

3. Katona P, Katona-Apte J. The interaction between nutrition and infection. Clin Infect Dis. 2008;46(10):1582-8 https://doi.org/10.1086/587658.

4. UNICEF. WHO, World Bank Group. Levels And Trends in Child Malnutrition in UNICEF/WHO/World Bank Group Joint Child Malnutrition Estimates Key 
Findings of the 2016 edition. New York: UNICEF; Geneva: WHO; Washington DC: World Bank.

5. Kandala NB, Madungu TP, Emina JB, Nzita KP, Cappuccio FP. Malnutrition among children under the age of five in the Democratic Republic of Congo (DRC): does geographic location matter? BMC Public Health. 2011;11:261.

6. Akombi BJ, Agho KE, Hall JJ, Merom D, Astell-Burt T, Renzaho AM. Stunting and severe stunting among children under-5 years in Nigeria: a multilevel analysis. BMC Pediatr. 2017;17:15.

7. Akombi BJ, Agho KE, Merom D, Hall JJ, Renzaho AM. Multilevel analysis of factors associated with wasting and underweight among children underfive years in Nigeria. Nutrients. 2017;9:44.

8. Medhin G, Hanlon C, Dewey M, Alem A, Tesfaye F, Worku B, Tomlinson M, Prince M. Prevalence and predictors of undernutrition among infants aged six and twelve months in Butajira, Ethiopia: the P-MaMiE Birth Cohort. BMC Public Health. 2010;10:27.

9. United Nations International Children's Fund. Multi-Sectoral Approaches to Nutrition. Nutrition-Specific and Nutrition-Sensitive Interventions to Accelerate Progress. New York: United Nations International Children's Fund; 2014.

10. Overview of the Health System in Rwanda. Available at https://dhsprogram. com/pubs/pdf/SPA3/02Chapter2.pdf. Accessed 19 Mar 2018.

11. Mustapher S. Kilindi seeks to reduce maternal deaths. Daily News. 2013, June 16. https://www.dailynews.co.tz/news/kilindi-seeks-to-reduce-maternaldeaths.aspx. Accessed 19 Mar 2018.

12. West-Slevin K, Barker C, Hickmann M. Snapshot: Tanzania's health system. Health Policy Project, Futures Group. 2015. Available at https://www. healthpolicyproject.com/pubs/803_TanzaniaHealthsystembriefFINAL.pdf

13. World Vision. Evaluation of World Vision's East Africa Maternal, Newborn and Child Health Project (EAMNeCH). Available at https://www.wvi.org/sites/ default/files/WV\%20EAMNeCH\%20Evaluation\%20Final.pdf. Accessed 5 Apr 2018.

14. Ministry Of Health. Health Sector Strategic Plan Promoting People's Health to enhance Social Economic Development (2010/11-2014/15). July 2010 [online] Available at http://www.health.go.ug/docs/HSSP_III_2010.pdf.

15. Agho KE, Mukabutera C, Mukazi M, Ntambara M, Mbugua I, Dowling M, Kamara JK. Moderate and severe household food insecurity predicts stunting and severe stunting among Rwanda children aged 6-59 months residing in Gicumbi district. Maternal \& child nutrition. 2018;13:e12767.

16. Geoffrey B, Gilbert K, Anita K. Bridging the gap for maternal newborn and child health human resources in rural Uganda: experiences and lessons learnt from world Vision east African maternal newborn and child health project implementation, Kitgum District. Int J Nur Care. 2017; 1(6):1-1.

17. World Vision International. Facilitator's guide to 7-11 health information. Available at https://www.wvi.org/health/publication/facilitators-guide-7-11health-strategy. Accessed 8 Mar 2018.

18. De Onis M, Onyango A, Borghi E, Siyam A, Pinol A, Garza C, Martines J, Martorell R, Victora CG, Bhan MK, Araújo CL. WHO child growth standards: length/height-for-age, weight-for-age, weight-for-length, weight-for-height and body mass index-for-age-Methods and development. WHO; 2006.

19. Filmer D, Pritchett $\mathrm{LH}$. Estimating wealth effects without expenditure data—or tears: an application to educational enrolments in states of India. Demography. 2001;38(1):115-32.

20. WHO, UNICEF. Progress on drinking water and sanitation: 2014 update. Geneva: World Health Organization; 2014.

21. Darteh EK, Acquah E, Kumi-Kyereme A. Correlates of stunting among children in Ghana. BMC Public Health. 2014;14(1):1.

22. Salami A, Kamara AB, Brixiova Z. Smallholder agriculture in East Africa: trends, constraints and opportunities. African Development Bank: Tunis; 2010.

23. Mwangome M, Prentice A, Plugge E, Nweneka C. Determinants of appropriate child health and nutrition practices among women in rural Gambia. J Health Popul Nutr. 2010;28(2):167.

24. Kavosi E, Hassanzadeh Rostami Z, Kavosi Z, Nasihatkon A, Moghadami M, Heidari $\mathrm{M}$. Prevalence and determinants of under-nutrition among children under six: a cross-sectional survey in Fars province, Iran. Int J Health Policy Manag. 2014;3:71-6.

25. Masiye F, Chama C, Chitah B, Jonsson D. Determinants of child nutritional status in Zambia: an analysis of a national survey. Zambia Soc Sci J. 2010;1(1):4.

26. Tiwari R, Ausman LM, Agho KE. Determinants of stunting and severe stunting among under-fives: evidence from the 2011 Nepal demographic and health survey. BMC Pediatr. 2014;14(1):1.
27. Akombi BJ, Agho KE, Hall JJ, Wali N, Renzaho AMN, Merom D. Stunting, wasting and underweight in sub-Saharan Africa: a systematic review. Int J Environ Res Public Health. 2017;14(8):E863.

28. Masibo PK, Makoka D. Trends and determinants of undernutrition among young Kenyan children: Kenya demographic and health survey; 1993, 1998, 2003 and 2008-2009. Public Health Nutr. 2012;15(09):1715-27.

29. Ramli AKE, Inder KJ, Bowe SJ, Jacobs J, Dibley MJ. Prevalence and risk factors for stunting and severe stunting among under-fives in North Maluku province of Indonesia. BMC Pediatr. 2009;9(1):1.

30. Shiratori S. Determinants of Child Malnutrition in Tanzania: a Quantile Regression Approach. 2014 Annual Meeting, July 27-29, 2014, Minneapolis, Minnesota (No. 170304). Agric Appl Econ Assoc. 2014.

31. Dabale GA, Sharma MK. Determinants of wasting among under-five children in Ethiopia: (a multilevel logistic regression model approach). Int J Stat Med Res. 2014:3:368.

32. Lesiapeto MS, Smuts CM, Hanekom SM, Du Plessis J, Faber M. Risk factors of poor anthropometric status in children under five years of age living in rural districts of the eastern cape and KwaZulu-Natal provinces, South Africa. S Afr J Clin Nutr. 2010;23:202-7.

33. Cumming O, Cairncross S. Can water, sanitation and hygiene help eliminate stunting? Current evidence and policy implications. Matern Child Nutr. 2016;12(Suppl 1):91-105

34. Asfaw M, Wondaferash M, Taha M, Dube L. Prevalence of undernutrition and associated factors among children aged between six to fifty nine months in Bule Hora district, South Ethiopia. BMC Public Health. 2015;15:41.

35. Black RE, Victora CG, Walker SP, Bhutta ZA, Christian P, de Onis M, Uauy R. Maternal and child undernutrition and overweight in low-income and middle-income countries. Lancet. 2013;382:427-51.

36. Rodriguez L, Cervantes E, Ortiz. Malnutrition and Gastrointestinal and Respiratory Infections in Children: A Public Health Problem. Int J Environ Res Public Health. 2011;8(4):1174-205 https://doi.org/10.3390/ijerph8041174.

37. Mwiru R, Spiegelman D, Hertzmark E, Duggan C, Msamanga G, Aboud S, Fawzi W. Nutritional predictors of acute respiratory infections among children born to HIV-infected women in Tanzania. J Trop Pediatr. 2013;59(3): 203-8 https://doi.org/10.1093/tropej/fmt004.

38. Geberetsadik A, Worku A, Berhane Y. Factors associated with acute respiratory infection in children under the age of 5 years: evidence from the 2011 Ethiopia demographic and health survey. Pediatr Health, Med and Therapeutics. 2015;6:9-13 https://doi.org/10.2147/PHMT.S77915.

39. Grant CC, Wall CR, Gibbons MJ, Morton SM, Santosham M, Black RE. Child nutrition and lower respiratory tract disease burden in New Zealand: a global context from a national perspective. J Paediatr Child Health. 2011;47:497-504.

40. World Health Organization, Unicef. Ending preventable child deaths from pneumonia and diarrhoea by 2025: The integrated Global Action Plan for Pneumonia and Diarrhoea (GAPPD).

Ready to submit your research? Choose BMC and benefit from:

- fast, convenient online submission

- thorough peer review by experienced researchers in your field

- rapid publication on acceptance

- support for research data, including large and complex data types

- gold Open Access which fosters wider collaboration and increased citations

- maximum visibility for your research: over $100 \mathrm{M}$ website views per year

At $\mathrm{BMC}$, research is always in progress.

Learn more biomedcentral.com/submission 